\title{
Effects of thermal annealing on the structure of ferroelectric thin films
}

\author{
Jiang-Li Cao ${ }^{\mathrm{a}} *$, Axel Solbach ${ }^{\mathrm{a}}$, Thomas Weirich ${ }^{\mathrm{b}}$, Joachim Mayer ${ }^{\mathrm{b}}$, Ulrich Böttger ${ }^{\mathrm{c}}$, Ulrich \\ Ellerkmann ${ }^{\mathrm{c}}$, Peter J. Schorn ${ }^{\mathrm{c}}$, Peter Gerber ${ }^{\mathrm{c}}$, Rainer Waser ${ }^{\mathrm{c}}$, and Uwe Klemradt ${ }^{\mathrm{a}}$ \\ a Physikalisches Institut B II, RWTH Aachen, Germany \\ b Gemeinschaftslabor für Elektronenmikroskopie, RWTH Aachen, Germany \\ c Institut für Werkstoffe der Elektrotechnik II, RWTH Aachen, Germany
}

\section{Abstract:}

Effects of thermal annealing on the structure of polycrystalline $\mathrm{Pb}\left(\mathrm{Zr}_{0.3} \mathrm{Ti}_{0.7}\right) \mathrm{O}_{3}(\mathrm{PZT})$ ferroelectric thin films prepared by chemical solution deposition (CSD) on $\mathrm{Pt} / \mathrm{TiO}_{\mathrm{x}}$ electrode stacks were studied using scanning electron microscopy (SEM), transmission electron microscopy (TEM) and grazing incidence X-ray specular and diffuse reflectivity of synchrotron radiation. The stratified multilayered structure and element diffusions in the sample were characterized by TEM. Global statistical structural parameters including the density, surface or interface roughness and thickness of each layer in the samples were obtained from fitting the Xray specular reflectivity using a homogeneous stratified multilayer model of $\mathrm{PZT} / \mathrm{Pt} / \mathrm{TiO} \mathrm{x}_{\mathrm{x}} / \mathrm{SiO}_{2}$. The results showed that the PZT surface and PZT/Pt interface roughness changed only slightly during thermal annealing in oxygen at $700^{\circ} \mathrm{C}$. By contrast, the density increase of the PZT ceramic and density decrease of the Pt bottom electrode during annealing were observed. A high density value of the PZT ceramic film after the annealing was found up to $99.8 \%$ of the theoretical value of the corresponding bulk ceramics. The density changes of the PZT and Pt layers were further confirmed by X-ray diffuse reflectivity. The influences of the annealing treatment on the density changes of the PZT and Pt layers were attributed to the further densification of the PZT ceramic and incorporation of light elements such as $\mathrm{Zr}$, Ti and $\mathrm{O}$ from 
the neighboring layers into the Pt layer respectively, as discussed in correlation with the TEM analyses.

Keywords: ferroelectric, lead zirconate titanate, X-ray reflectivity, synchrotron radiation, thin films

* Corresponding authors.

Present contact address:

L2 - 140

School of Mechanical, Materials and Manufacturing Engineering

The University of Nottingham

Nottingham NG7 2RD

UK

Tel: $0044(0) 1159513920$

Fax: $0044(0) 1159513800$

E-mail: $\underline{\text { Cao@physik.rwth-aachen.de }}$ 


\section{Introduction}

Owing to their promising applications in non-volatile random access memories and micro-electromechanical systems, ferroelectric thin films have received much attention. These ferroelectric devices typically have a sandwiched nano-multilayered structure, i.e. top electrode / ferroelectric film / bottom electrode with the thickness of each layer on the nanometer scale. The two-dimensional films are deposited on a substrate, which is generally a Si wafer. Because of the complex structure, the electrical properties of such nano-multilayered devices are not only very sensitive to the properties of each individual layer but depend also on the interfacial transitions, both being strongly influenced by the fabrication process ${ }^{[1,2]}$. Thus comprehensive structural characterizations of the ceramic thin films including buried interfaces are of crucial importance for a better understanding of the relationship between the structural and electrical properties of miniaturized ferroelectric devices.

Various advanced analytical techniques have been adopted to characterize the structural properties of ferroelectric thin films, such as scanning probe microscopy (SPM) for domain switching studies using various force interactions ${ }^{[3]}$, and high-resolution transmission electron microscopy (HRTEM) for structural investigations of the surface or buried interfaces ${ }^{[4]}$. Recently, the potential of synchrotron radiation has been more extensively exploited for the structural studies of the ferroelectric and dielectric thin film devices. For example, S. Kimura et al studied the fatigue mechanism of polycrystalline $\mathrm{Pb}\left(\mathrm{Zr}_{\mathrm{x}} \mathrm{Ti}_{1-\mathrm{x}}\right) \mathrm{O}_{3}$ films with asymmetric metal electrodes by using X-ray diffraction, from which they observed a broadening of the $(00 l)$ plane spacing with the polarization fatigue ${ }^{[5]}$. S.J. van Reeuwijk et al reported their X-ray diffraction studies on the domain switching from $(h k l)$ to $(h k \bar{l})$ of $\mathrm{BaTiO}_{3}$ thin films on $\mathrm{Nb}$ doped $\mathrm{SrTiO}_{3}$ substrate ${ }^{[6]}$. 
In this work, the impact of thermal annealing on $\mathrm{Pb}\left(\mathrm{Zr}_{0.3} \mathrm{Ti}_{0.7}\right) \mathrm{O}_{3}$ ferroelectric thin films on $\mathrm{Pt} / \mathrm{TiO}_{\mathrm{x}}$ electrode stacks is studied by a combination of complementary analytical tools. $\mathrm{Pb}\left(\mathrm{Zr}_{\mathrm{x}} \mathrm{Ti}_{1-\mathrm{x}}\right) \mathrm{O}_{3}(\mathrm{PZT})$ thin films are considered as a model system because of their high remanent polarization, relatively low fabrication temperature and adjustable composition. Scanning electron microscopy (SEM) is used for the surface morphology observation, whereas transmission electron microscopy (TEM) is employed for the cross-sectional observation and element mapping. Particular emphasis is given to specular and non-specular X-ray reflectivity (XRR), which are standard tools for the non-destructive structural analysis of crystalline and amorphous multilayers in many areas of research, but which seem to have received little attention in the ferroelectrics community so far. Specifically, we demonstrate the ability of XRR to monitor even minute density changes, in addition to providing information on the film thickness and root mean square (rms) interface roughness. The use of highly intense and strongly collimated synchrotron radiation also allowed us to access electrode layers buried deeply beneath a relatively thick PZT film.

\section{Experimental}

\subsection{Sample preparation}

$\mathrm{Pb}\left(\mathrm{Zr}_{0.3} \mathrm{Ti}_{0.7}\right) \mathrm{O}_{3}$ thin films were prepared on standard commercial Si wafers through the following steps. Firstly, a silicon wafer with a size of $25.4 \mathrm{~mm} \times 25.4 \mathrm{~mm} \times 0.6 \mathrm{~mm}$ was thermally oxidized to form an amorphous $\mathrm{SiO}_{2}$ of about 300nm thick. Secondly, a Ti adhesion layer was deposited by sputtering and subsequently oxidized at $700^{\circ} \mathrm{C}$ in a pure oxygen atmosphere for 5min. Thirdly, a $120 \mathrm{~nm}$ thick Pt layer was deposited by sputtering on the Ti oxide layer at $200^{\circ} \mathrm{C}$ and subsequently crystallized at $700^{\circ} \mathrm{C}$ in oxygen for $5 \mathrm{~min}$. Then the PZT film was prepared using a chemical solution deposition by spin-coating method ${ }^{[7]}$. After each layer of the PZT film 
was spin-coated, the sample was dried in air at $200^{\circ} \mathrm{C}$ for $2 \mathrm{~min}$ and pyrolyzed at $400^{\circ} \mathrm{C}$ for $2 \mathrm{~min}$. After the deposition of each three layers, the sample was crystallized at $700^{\circ} \mathrm{C}$ in oxygen for $10 \mathrm{~min}$. It is compulsory to repeat this procedure in order to relax the stress during the fabrication until the PZT films have reached their intended thickness. In this study the PZT films were formed by the deposition of three layers. Then two long narrow samples of $25.4 \mathrm{~mm} \times 5 \mathrm{~mm}$ were cut out from the center of the Si wafer. One was designated as unannealed while the other one that received an additional annealing at $700^{\circ} \mathrm{C}$ for $5 \mathrm{~min}$ was designated as annealed.

\subsection{Analytical techniques}

SEM was used for surface morphology observation using a Zeiss Gemini DSM 982 microscope operated at $4 \mathrm{kV}$. An annealed PZT thin film sample on another Si substrate was used for TEM analyses using a Philips TECNAI FEI 20 microscope with an energy filter (Gatan GIF 2000), an energy dispersive X-ray (EDX) detector and a high angle annular dark field (HAADF) detector. A thin TEM cross-sectional lamellar with a thickness less than $100 \mathrm{~nm}$ was prepared by focused ion beam (FIB) cutting on a FEI STRATA FIB 205 ion beam system.

X-ray reflectivity measurements were carried out with synchrotron radiation from Hasylab (DESY) using a high-resolution reflectometer. The experiments were performed at the bending magnet beamline E2 with a $\mathrm{Si}(111)$ double monochromator tuned to $11 \mathrm{keV}(\lambda=$ $1.127 \AA)$. In addition to the measurements along the specular path $(\theta-2 \theta$ scan $)$, two different non-specular scan types were performed: offset scans at a deliberately detuned specular condition $\left(\alpha_{i}-\alpha_{f}= \pm 2 \varepsilon\right.$, where $\alpha_{i}$ is the incident angle, $\alpha_{f}$ the exit angle and $\varepsilon$ the offset angle) and detector scans at a fixed angle of incidence angle $\left(\alpha_{i}=0.6000^{\circ}\right)$. The incident beam had a height of $75 \mu \mathrm{m}$ in the scattering plane and a width of $2 \mathrm{~mm}$. A photodiode detector was 
used for the measurements at low angles while a $\mathrm{NaI}$ detector was used for the higher angle measurements. The measured specular intensities were corrected for the extrinsic background (dark currents etc.) as well as for the intrinsic background (diffuse scattering in specular direction), which was determined by two offset scans adjacent to the specular path in reciprocal space. Finally, the specular reflectivities obtained in this way were corrected for illumination at low angles, where the footprint of the beam exceeded the sample size. The experimental data were fitted by slab calculations based on the Parratt algorithm ${ }^{[8]}$.

It should be mentioned that X-ray specular reflectivity can be understood as classical Fresnel reflectivity, provided the proper index of refraction for X-rays is used. Therefore, the specular X-ray reflectivity is sensitive to the electron density profile normal to the sample surface, and insensitive to whether the layers are amorphous, crystalline or even liquid since no diffraction is involved. The structural parameters of each layer including the thickness, interfacial roughness and density can thus be derived. It should be noted that these parameters correspond to the sample properties associated with the growth direction; these are consequently probed by a momentum transfer perpendicular to the sample surface. By contrast, in the diffuse reflectivity the momentum transfer has a component parallel to the sample surface, allowing the access to the height-height correlation function of rough interfaces.

\section{Results}

\subsection{Surface and interface analyses using SEM and TEM}

Fig. 1 shows a SEM image of the surface morphology of the annealed PZT thin film. The PZT ceramic layer consists of very fine grains and is very dense. No void or crack can be observed. 
The dark field (DF) TEM cross-sectional image in Fig. 2(a) shows clearly the multilayered structure and the interface quality in the PZT thin film sample. From top to bottom the layers are the PZT, Pt, $\mathrm{TiO}_{x}, \mathrm{SiO}_{2}$ and $\mathrm{Si}$ substrate, respectively. Fig. 2(b) shows the element mappings in the cross-sectional profile of the multilayered structure measured using scanning transmission electron microscopy (STEM). From top to bottom the images are the HAADF cross-sectional image of the observed area, the corresponding $\mathrm{Pb}, \mathrm{Zr}, \mathrm{Ti}, \mathrm{O}$ and $\mathrm{Pt}$ mapping, respectively. $\mathrm{Pb}, \mathrm{Zr}$, $\mathrm{Ti}$ and $\mathrm{O}$ can all be found in the $\mathrm{Pt}$ electrode, which reveal the element diffusions during the fabrication process of the sample. By contrast, the diffusion of Pt is not observable.

\subsection{X-ray specular reflectivity}

X-ray specular reflectivity curves of the unannealed and annealed PZT thin film samples are plotted on a logarithmic scale in Fig. 3(a) and (b) as a function of $q_{z}=4 \pi \times \sin \theta / \lambda$, where $q_{z}$ is the momentum transfer perpendicular to the sample surface, $\theta$ the grazing incidence angle and $\lambda$ the $\mathrm{X}$-ray wavelength. The initial flat part of each reflectivity curve is the total reflection region of PZT. With increasing $q_{z}$ (or equivalently, the incidence angle), the reflectivity decreases rapidly and the X-ray beams start to penetrate into the ceramic layer. The oscillations appearing between $0.05 \AA^{-1}$ and $0.08 \AA^{-1}$ are caused by the interference of two beams; one reflected from the buried PZT/Pt interface and the other from the PZT surface, indicating the well-defined PZT/Pt interfaces for both samples. Around $q_{z}=0.08 \AA^{-1}$, the reflectivity decreases again and some oscillations with different shape appear, which result from the penetration of the X-ray beam into the Pt bottom electrode, giving rise to more complicated interferences among the various reflected beams from each interface of the multilayer. Since the electron density of Pt 
exceeds significantly that of PZT, the first intensity drop in the reflectivity curve with the increase of $q_{z}$ essentially reveals the critical angle of the PZT ceramics, while the second intensity drop reveals the critical angle of the bottom Pt electrode. The PZT critical-angle regions in the two curves are plotted on a linear scale in the inset of Fig. 3(b) for comparison.

The two experimental reflectivity curves are fitted using the program PCTRF, as shown in Fig. 3(a) and (b) (solid lines), respectively ${ }^{[9]}$. Because the momentum transfer occurs normal to the sample surface and the reflectivity becomes sensitive to the vertical average electron density profile, only the structural information is relevant including the thickness, density of each layer, as well as the surface and interface roughness between two adjacent layers. The roughness obtained here is a root-mean square (rms) roughness characterizing the perpendicular electron density fluctuations of a surface or interface, assuming that the fluctuations meet a Gaussian distribution according to the model of Névot and Croce. For the fits, a reasonably simple structural model is assumed in order to minimize the number of free parameters. The multilayer is described as a stack of homogeneous $\mathrm{PZT} / \mathrm{Pt} / \mathrm{TiO}$ x layers on a semi-infinite $\mathrm{SiO}_{2}$ substrate, owing to the large thickness of the $\mathrm{SiO}_{2}$ layer. Previously we have determined that the $\mathrm{TiO}_{\mathrm{x}}$ composition is slightly deviated from stoichiometric $\mathrm{TiO}_{2}{ }^{[10]}$. Here $\mathrm{Ti}_{8} \mathrm{O}_{15}$ is used for the fits for approximation. From the fits, PZT densities of 7.97 and $8.01 \mathrm{~g} / \mathrm{cm}^{3}$ for the unannealed and annealed samples are derived which correspond respectively to $99.3 \%$ and $99.8 \%$ of the theoretical density of $8.03 \mathrm{~g} / \mathrm{cm}^{3}$ calculated using the lattice parameters of bulk ceramics reported earlier ${ }^{[11]}$. The PZT/Pt interfacial roughness does not appear to increase. The density of the Pt bottom electrode decreases from $20.6 \mathrm{~g} / \mathrm{cm}^{3}$ to $20.3 \mathrm{~g} / \mathrm{cm}^{3}$, in agreement with the Pt layer expansion of $2.5 \%$. The structural characteristics of the two series of PZT thin films are summarized in Table 1, in which the error margins are also given. Here it should be noted that 
the inset of Fig. 3(b) provides clear experimental evidence for a density difference between the two PZT density values although the error bars determined from the independent fits on a log scale to the specular reflectivity curves do overlap. A relative density difference, $\Delta \rho / \rho=\Delta \delta / \delta=2 \Delta q_{z} / q_{z}=(0.92 \pm 0.15) \%$, can be determined directly from the shift since the critical angle of total reflection for X-rays is given by $\alpha_{c}=\sqrt{2 \delta}$, where $\delta=\lambda^{2} \rho_{e l} r_{0} / 2 \pi=1-n, \lambda$ is the X-ray wavelength, $\rho_{e l}$ the electron density of the matter, $r_{0}$ the classical electron radius and $n$ the refractive index of the matter.

\subsection{X-ray diffuse reflectivity}

Detector scans with the unannealed and annealed samples were measured in order to verify the critical-angle shift of the PZT and Pt layers upon annealing. In this geometry, there will be peaks in the diffuse reflectivity known as Yoneda scatterings or anomalous reflections when the exit angle equals the critical angles where the electric field is at its maximum value ${ }^{[12-}$ 14].

Fig. 4 displays the detector scans of the two samples in which the incidence angle was kept at $0.6000^{\circ}$ while the detector was rotated. In each curve, the first two peaks are Yoneda peaks whose positions correspond to the critical angles of the PZT ceramic and Pt, respectively, while the third peak corresponds to the specular reflection. Accordingly, the critical angles of PZT and Pt before and after the annealing are determined to be $0.2207 \pm 0.0034^{\circ}$ and $0.3967 \pm 0.0011^{\circ}, 0.2284 \pm 0.0036^{\circ}$ and $0.3929 \pm 0.0009^{\circ}$. The density changes of Pt and PZT upon annealing observed from specular reflectivity are confirmed by the corresponding Yoneda peak shifts in the diffuse reflectivity. Additionally, the intensity oscillations observed in specular reflectivity are absent here, especially at lower angles, indicating that a correlation of interface 
roughness among the constituent layers in the growth direction was not established during the film growth.

\section{Discussion}

In the present study, a combination of analytical techniques including SEM, TEM and Xray reflectivity of synchrotron radiation were employed to investigate the effects of thermal annealing on the structure of the PZT thin films on $\mathrm{Pt} / \mathrm{TiO}_{\mathrm{x}}$ electrode stacks. Through STEM element mapping analysis the diffusions of elements including $\mathrm{Pb}, \mathrm{Zr}$, $\mathrm{Ti}$ and $\mathrm{O}$ into the $\mathrm{Pt}$ layer were observed. The element diffusions can be attributed to the high temperature treatments during the fabrication processing of the samples. Using X-ray reflectivity, global statistical information including the thickness, density, surface and interface roughness of each constituent layer in the samples were obtained from the fits using a structure model of $\mathrm{PZT} / \mathrm{Pt} / \mathrm{TiO}_{\mathrm{x}} / \mathrm{SiO}_{2}$.

The roughness of PZT ceramic surface, $\mathrm{PZT} / \mathrm{Pt}$ interface and $\mathrm{Pt} / \mathrm{TiO}_{\mathrm{x}}$ interface were rather stable during annealing. The increase of the PZT density proves the further densification of the ceramic layer and indicates the improvement of its microstructure upon annealing. The densification of PZT will eliminate defects like pores and voids in the ceramic film and therefore improve the structural properties. Furthermore, it is interesting that the density and thickness of the Pt bottom electrode changed during annealing. The density decrease of Pt from $20.6 \mathrm{~g} / \mathrm{cm}^{3}$ to $20.3 \mathrm{~g} / \mathrm{cm}^{3}$ can be ascribed to the incorporation of light elements such as $\mathrm{Zr}$, Ti and $\mathrm{O}$ during the fabrication process according to the STEM analysis. In agreement with the Pt density decrease, the thickness of the Pt layer was found to increase upon annealing. It is reasonable to expect that the expansion of the Pt layer and the densification of PZT will occur during the high temperature treatment as an intrinsic aspect of the electrode stack in the current fabrication process. 
An important related issue is the effect of thermal annealing on the electrical properties of the PZT thin film as the observed structural changes indicate. High thermal stability of the ferroelectric devices during the fabrication process is required since the devices need to withstand a certain thermal budget. Although a higher densification of PZT ceramic film is obtained and the structural defects are reduced correspondingly, the effect of the thermal treatment on the electrical properties of PZT ferroelectric thin films needs to be examined carefully. Normally, for CSD-derived PZT on Si wafer, the stress in the ceramic films is tensile due to the thermal mismatch which is caused intrinsically by the lower thermal expansion coefficient of Si compared to those of PZT ceramics and Pt ${ }^{[15]}$. Because of the metal electrode expansion and the ceramic layer shrinkage, there will be an additional tensile stress component superimposed on the PZT ceramics owing to the clamping effects of the electrode layer. This stress component is caused by the structural changes during high temperature annealing.

The domain structure of ferroelectric ceramics depends to a great extent on the stress conditions prevailing when the Curie temperature is crossed, or even changes in the stress conditions at room temperature. Previously it was reported that the volume fraction of a-type domains increased when substrates with smaller thermal expansion coefficients were used for PZT deposition and the PZT films were placed under tensile stress ${ }^{[16]}$. M.B. Kleman et al reported the switching of elastic domains in PZT thin films in order to relax the external strain applied at room temperature ${ }^{[17]}$, which reveals the important role of the mechanical boundaries in determining the electrical properties of the ferroelectric devices. In the present study, the $\mathrm{PZT} / \mathrm{Pt}$ interaction becomes complicated by the increase of the dissimilarity between the ceramic and electrode, as suggested by the results obtained. Thus, it is likely that the structure of the ceramic layer will be affected under the stress as well as the electrical properties of the resulting 
devices. Moreover, the effects of the thermal treatment may become more complicated when the PZT films are constructed layer by layer and subsequently each layer has a different annealing history, therefore a gradient structure in thicker PZT films can be expected.

\section{Conclusions}

In summary, as a model system $\mathrm{PZT}$ thin films on bilayer $\mathrm{Pt} / \mathrm{TiO}_{\mathrm{x}}$ electrode stacks were derived by a chemical solution deposition, and were characterized by SEM and TEM as well. Grazing incidence X-ray specular and diffuse reflectivity of synchrotron radiation was employed for the detailed structural investigation of ferroelectric thin film devices.

1. Through element mapping using STEM, the incorporations of $\mathrm{Pb}, \mathrm{Zr}$, Ti and $\mathrm{O}$ into the Pt electrode were observed which were attributed to the thermal diffusion during the fabrication process of the sample.

2. Statistical structural information of the nano-multilayered samples was derived from X-ray specular reflectivity which reveals a further densification of the PZT ceramics and an expansion of the Pt electrode in the polycrystalline PZT thin film sample during the annealing treatment. The PZT surface roughness and PZT/Pt interface roughness changed only slightly.

3. The Pt density decrease was attributed to the incorporation of the light elements into the Pt layer during annealing, while the PZT density increase is due to the further densification of the ceramic layer.

4. No vertical roughness replication in the multilayered structure was observed through X-ray detector scans.

Acknowledgements: Dr. Jiang-Li Cao thanks the Alexander von Humboldt Foundation for the support. The authors are grateful for the support of DESY for the project grant II-03-009. 


\section{References:}

1. J.E. Lim, D.Y. Park, J.K. Jeong, G. Darlinski, H.J. Kim, C.S. Hwang, S.H. Kim, C.Y. Koo, H.J. Woo, D.S. Lee and J. Ha, "Dependence of ferroelectric performance of sol-gel-derived $\mathrm{Pb}(\mathrm{Zr}, \mathrm{Ti}) \mathrm{O}_{3}$ thin films on bottom-Pt-electrode thickness,” Appl. Phys. Lett., 81[17], 3224$3226(2002)$.

2. S. Okamura, N. Abe, Y. Otani and T. Shiosaki, "Influence of $\mathrm{Pt} / \mathrm{TiO}_{2}$ bottom electrodes on the properties of ferroelectric $\mathrm{Pb}(\mathrm{Zr}, \mathrm{Ti}) \mathrm{O}_{3}$ thin films," Integrated Ferroelectrics, 52, 127-136 (2003).

3. A. Gruverman, H. Tokumoto, A.S. Prakash, S. Aggarwal, B. Yang, M. Wuttig, R. Ramesh, O. Auciello and T. Venkatesan, "Nanoscale imaging of domain dynamics and retention in ferroelectric thin films,” Appl. Phys. Lett., 71[24], 3492-3494 (1997).

4. M.W. Chu, I. Szafraniak, R. Scholz, C. Harnagea, D. Hesse, M. Alexe and U. Gosele, "Impact of misfit dislocations on the polarization instability of epitaxial nanostructured ferroelectric perovskites ," Nature Materials, 3, 87-90 (2004).

5. S. Kimura, K. Izumi and T. Tatsumi, Tetragonal distortion of $\mathrm{c}$ domains in fatigued $\mathrm{Pb}(\mathrm{Zr}, \mathrm{Ti}) \mathrm{O}_{3}$ thin films determined by X-ray diffraction measurements with highly brilliant synchrotron radiation, Appl. Phys. Lett., 80[13], 2365-2367 (2002).

6. S.J. van Reeuwijk, K. Karakaya, H. Graafsma and S. Harkema, Polarization switching in $\mathrm{BaTiO}_{3}$ thin films measured by X-ray diffraction exploiting anomalous dispersion, J. Appl. Crystallogr., 37, 193-199 (2004).

7. C. Kuegeler, P. Gerber, U. Boettger and R. Waser, Thickness dependence of piezoelectric properties for PZT thin films with regard to MEMS applications, Integrated Ferroelectrics, 54, 527-535 (2003). 
8. L.G. Parratt, Surface studies of solids by total reflection of X-rays, Physical Review, 95, 359-369 (1954).

9. U. Klemradt, PCTRF Multilayer Reflectivity Fitting Program (available on request).

10. J.-L. Cao, A. Solbach, U. Böttger, P. J. Schorn, T. Schneller, R. Waser, T. Weirich, J. Mayer, H. Horn-Solle and U. Klemradt, Structural investigations of Pt/TiOx electrode stacks for ferroelectric thin film devices, (submitted).

11. N. Tohge, S. Takahashi and T. Minami, Preparation of $\mathrm{PbZrO}_{3}-\mathrm{PbTiO}_{3}$ ferroelectric thin films by the sol-gel process, J. Am. Ceram. Soc., 74, 67 (1991).

12. S.K. Sinha, E.B. Sirota and S. Garoff, "X-ray and neutron scattering from rough surfaces", Physical Review B, 38, 2297-2311 (1988).

13. Y. Yoneda, “Anomalous surface reflection of X-rays", Physical Review, 131, 2010-2013 (1963).

14. J. Daillant and A. Gibaud (eds.), X-ray and neutron reflectivity: principles and applications, Springer 1999.

15. R. Waser (ed.), Nanoelectronics and Information Technology; pp. 73, Wiley-VCH, Berlin 2003.

16. B. A. Tuttle, T. J. Garino, J. A. Voigt, T. J. Headley, D. Dimos, and M. O. Eatough, in Science and Technology of Electroceramic Thin Films, edited by O. Auciello and R. Waser (Kluwer Academic, The Netherlands, 1995).

17. M.B. Kelman and P.C. McIntyre, B.C. Hendrix, S.M. Bilodeau and J.F. Roeder, "Effect of applied mechanical strain on the ferroelectric and dielectric properties of $\mathrm{PbZr}_{0.35} \mathrm{Ti}_{0.65} \mathrm{O}_{3}$ thin films", J. Appl. Phys., 93(11), 9231-9236 (2003). 


\section{Captions:}

Fig. 1 SEM image of the PZT ceramic surface of the annealed sample.

Fig. 2(a) TEM dark field cross-sectional image of the $\mathrm{PZT} / \mathrm{Pt} / \mathrm{TiO}_{\mathrm{x}} / \mathrm{SiO}_{2} / \mathrm{Si}$ multilayered structure and (b) HAADF image of the cross-section of the sample and the corresponding element mappings of $\mathrm{Pb}, \mathrm{Zr}, \mathrm{Ti}, \mathrm{O}$ and $\mathrm{Pt}$ measured using STEM. In the element mappings, the brightness is proportional to the content of the element in that area.

Fig. 3 Specular reflectivity curves (open circles) and best fits (within a restricted model, solid lines) to the experimental data: (a) the unannealed sample and (b) the annealed sample. A comparison between the PZT critical-angle regions in the two specular reflectivity curves is shown in the inset of Fig. 3(b).

Fig. 4 Detector scans of the unannealed and annealed PZT thin film samples with an incidence angle of $0.6000^{\circ}$, from which the critical-angle shifts of PZT and Pt are confirmed.

Table 1 Structural parameters of the unannealed and annealed samples obtained from best fits to the specular reflectivity curves. 
Fig. 1 (Authors: Jiang-Li Cao et al)

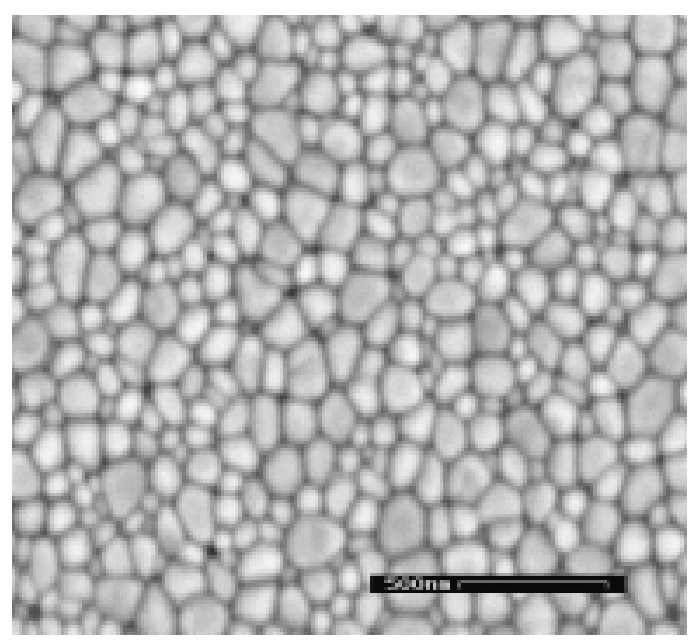


Fig. 2(a) (Authors: Jiang-Li Cao et al)

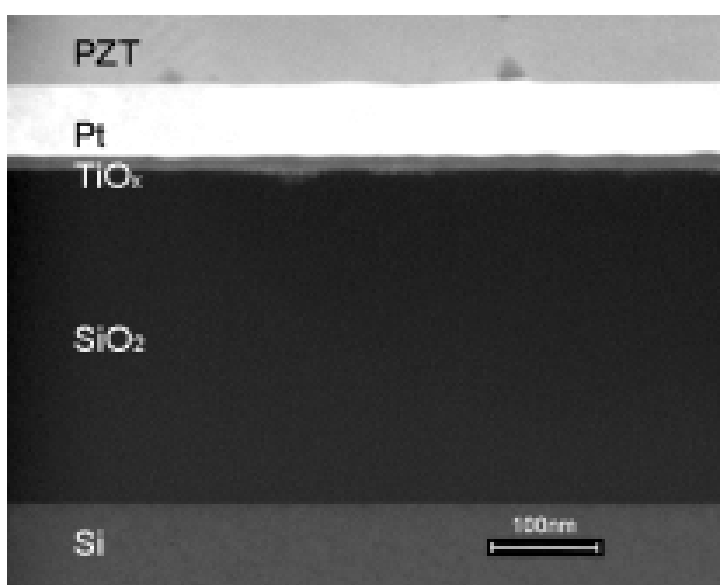


Fig. 2(b) (Authors: Jiang-Li Cao et al)

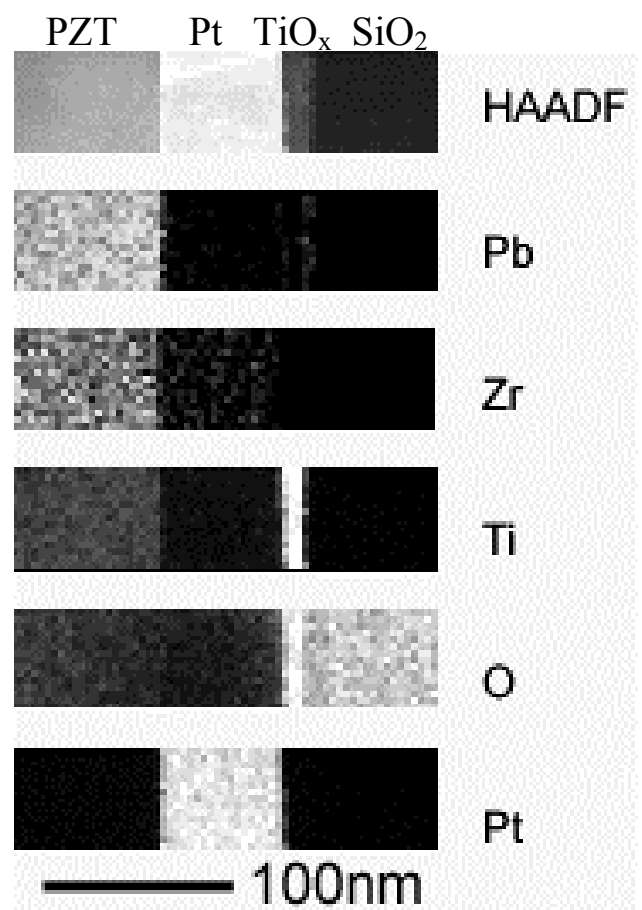


Fig. 3(a) (Authors: Jiang-Li Cao et al)

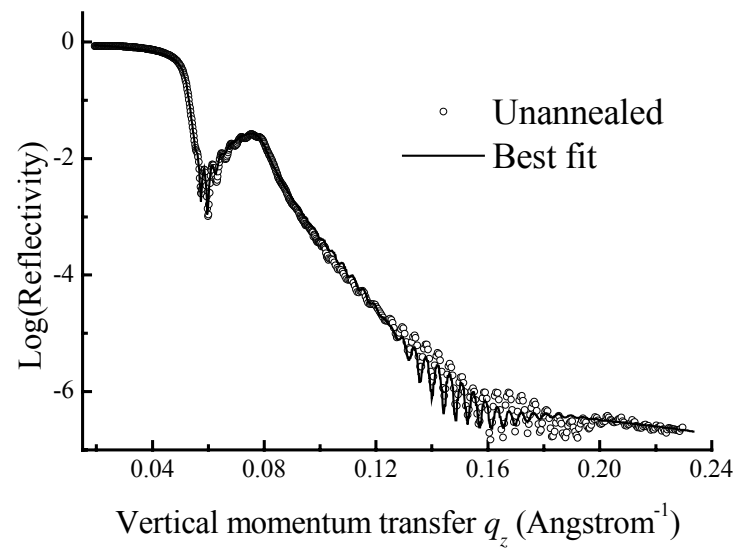


Fig. 3(b) (Authors: Jiang-Li Cao et al)

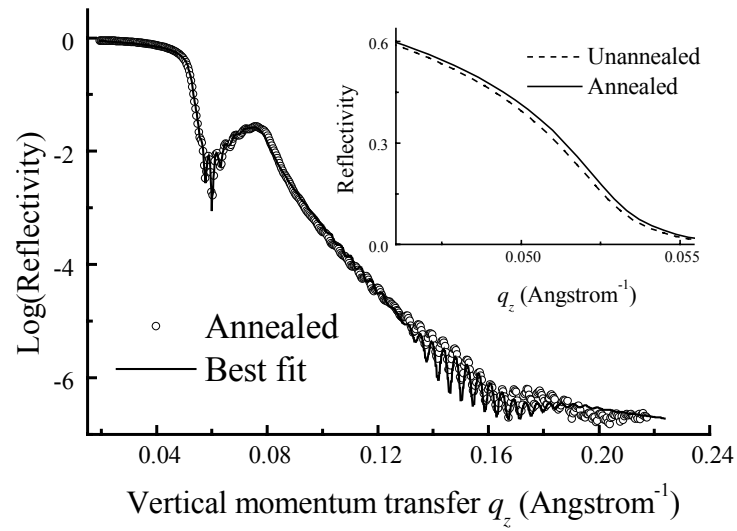


Fig. 4 (Authors: Jiang-Li Cao et al)

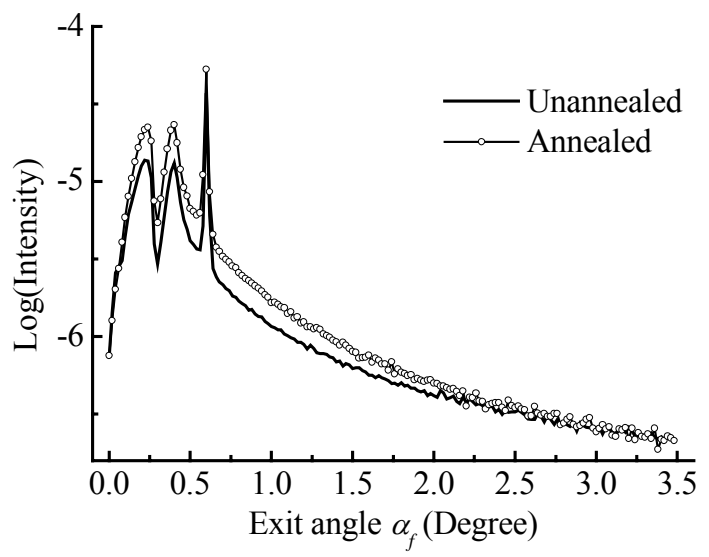


Table 1 (Authors: Jiang-Li Cao et al)

\begin{tabular}{|c|c|c|}
\hline Structure parameters & Unannealed sample & Annealed sample \\
\hline PZT surface roughness, $\sigma(\AA)$ & $54 \pm 1$ & $51 \pm 1$ \\
\hline PZT density, $\rho\left(\mathrm{g} / \mathrm{cm}^{3}\right)$ & $7.97 \pm 0.03$ & $8.01 \pm 0.03$ \\
\hline PZT thickness, $d(\AA)$ & $1064 \pm 8$ & $1050 \pm 8$ \\
\hline $\mathrm{PZT} / \mathrm{Pt}$ roughness, $\sigma(\AA)$ & $21.0 \pm 0.5$ & $20.2 \pm 0.5$ \\
\hline Pt density, $\rho\left(\mathrm{g} / \mathrm{cm}^{3}\right)$ & $20.6 \pm 0.2$ & $20.3 \pm 0.2$ \\
\hline Pt thickness, $d(\AA)$ & $1152 \pm 4$ & $1181 \pm 5$ \\
\hline $\mathrm{Pt} / \mathrm{TiO}_{\mathrm{x}}$ roughness, $\sigma(\AA)$ & $20 \pm 1$ & $18 \pm 1$ \\
\hline $\mathrm{TiO}_{\mathrm{x}}$ density, $\rho\left(\mathrm{g} / \mathrm{cm}^{3}\right)$ & $4.2 \pm 0.1$ & $4.2 \pm 0.1$ \\
\hline $\mathrm{TiO}_{\mathrm{x}}$ thickness, $d(\AA)$ & $134 \pm 3$ & $130 \pm 3$ \\
\hline $\mathrm{TiO}_{\mathrm{x}} / \mathrm{SiO}_{2}$ roughness, $\sigma(\AA)$ & $5.0 \pm 0.5$ & $5.4 \pm 0.5$ \\
\hline $\mathrm{SiO}_{2}$ density, $\rho\left(\mathrm{g} / \mathrm{cm}^{3}\right)$ & 2.2 & 2.2 \\
\hline
\end{tabular}

